\title{
Prediction of epileptic seizures based on Multivariate Multiscale Modified-Distribution Entropy
}

Si Thu Aung ${ }^{1}$, Yodchanan Wongsawat ${ }^{\text {Corresp. } 1}$

${ }^{1}$ Department of Biomedical Engineering, Faculty of Engineering, Mahidol University, Phuttamonthon, Nakornpathom, Thailand

Corresponding Author: Yodchanan Wongsawat

Email address: yodchanan.won@mahidol.ac.th

Epilepsy is a common neurological disease that affects a wide range of the world population and is not limited by age. Moreover, seizures can occur anytime and anywhere because of the sudden abnormal discharge of brain neurons, leading to malfunction. The seizures of approximately $30 \%$ of epilepsy patients cannot be treated with medicines or surgery; hence these patients would benefit from a seizure prediction system to live normal lives. Thus, a system that can predict a seizure before its onset could improve not only these patients' social lives but also their safety. Numerous seizure prediction methods have already been proposed, but the performance measures of these methods are still inadequate for a complete prediction system. Here, a seizure prediction system is proposed by exploring the advantages of multivariate entropy, which can reflect the complexity of multivariate time series over multiple scales (frequencies), called multivariate multiscale modified-distribution entropy (MM-mDistEn), with an artificial neural network (ANN). The phase-space reconstruction and estimation of the probability density between vectors provide hidden complex information. The multivariate time series property of MM-mDistEn provides more understandable information within the multichannel data and makes it possible to predict of epilepsy. Moreover, the proposed method is tested with two different analyses: simulation data analysis proves that the proposed method has strong consistency over the different parameter selections, and the results from experimental data analysis show that the proposed entropy combined with an ANN obtains performance measures of $98.66 \%$ accuracy, $91.82 \%$ sensitivity, $99.11 \%$ specificity, and 0.84 area under the curve (AUC) value. In addition, the seizure alarm system was applied as a postprocessing step for prediction purposes, and a false alarm rate of 0.014 per hour and an average prediction time of 26.73 minutes before seizure onset were achieved by the proposed method. Thus, the proposed entropy as a feature extraction method combined with an ANN can predict the ictal state of epilepsy, and the results show great potential for all epilepsy patients. 


\title{
1 Prediction of Epileptic Seizures Based on Multivariate
}

2 Multiscale Modified-Distribution Entropy

3

4

5

6

7

8

9

10

11

12

\author{
Si Thu Aung, Yodchanan Wongsawat
}

Department of Biomedical Engineering, Faculty of Engineering, Mahidol University, 25/25

Phuttamonthon 4 Road, Salaya, Nakhon Pathom 73170 Thailand

Corresponding Author:

Yodchanan Wongsawat

Department of Biomedical Engineering, Faculty of Engineering, Mahidol University, 25/25

Phuttamonthon 4 Road, Salaya, Nakhon Pathom 73170 Thailand

Email address: yodchanan.won@mahidol.ac.th

\section{Abstract}

Epilepsy is a common neurological disease that affects a wide range of the world population and is not limited by age. Moreover, seizures can occur anytime and anywhere because of the sudden abnormal discharge of brain neurons, leading to malfunction. The seizures of approximately $30 \%$ of epilepsy patients cannot be treated with medicines or surgery; hence these patients would benefit from a seizure prediction system to live normal lives. Thus, a system that can predict a seizure before its onset could improve not only these patients' social lives but also their safety. Numerous seizure prediction methods have already been proposed, but the performance measures of these methods are still inadequate for a complete prediction system. Here, a seizure prediction system is proposed by exploring the advantages of multivariate entropy, which can reflect the complexity of multivariate time series over multiple scales (frequencies), called multivariate multiscale modified-distribution entropy (MM-mDistEn), with an artificial neural network (ANN). The phase-space reconstruction and estimation of the probability density between vectors provide hidden complex information. The multivariate time series property of MM-mDistEn provides more understandable information within the multichannel data and makes it possible to predict of epilepsy. Moreover, the proposed method is tested with two different analyses: simulation data analysis proves that the proposed method has strong consistency over the different parameter selections, and the results from experimental data analysis show that the proposed entropy combined with an ANN obtains performance measures of $98.66 \%$ accuracy, $91.82 \%$ sensitivity, $99.11 \%$ specificity, and 0.84 area under the curve (AUC) value. In addition, the seizure alarm system was applied as a postprocessing step for prediction purposes, and a false alarm rate of 0.014 per hour and an average prediction time of 26.73 minutes before seizure onset were achieved by the proposed method. Thus, the proposed 
39

40

41

42

43

44

45

46

47

48

49

50

51

52

53

54

55

56

57

58

59

60

61

62

63

64

65

66

67

68

69

70

71

72

73

74

75

76

77

78

entropy as a feature extraction method combined with an ANN can predict the ictal state of epilepsy, and the results show great potential for all epilepsy patients.

Keywords ANN, distribution entropy, EEG, entropy, and epilepsy

\section{Introduction}

Epilepsy is one of the most common neurological disorders of the nervous system, affecting approximately 50 million people worldwide, and approximately 5 million people are diagnosed with epilepsy each year (WHO, 2019). Therefore, the social and economic impacts on patients with epilepsy are becoming increasingly concerning. Although the seizures of $70 \%$ of epileptic patients can be controlled by antiseizure medicines, the seizures of $30 \%$ of patients with epilepsy cannot be treated by either medicines or surgery; therefore, these patients must live their whole lives with epilepsy, and their seizures can occur anytime and anywhere (Fujiwara et al., 2015). Electroencephalogram (EEG) can record the patients' brain activities and be used as a tool for diagnosing and analyzing epilepsy (Wang et al., 2010). Thirty percent of epileptic patients whose seizures cannot be controlled urgently need a system that can improve their lives, by successfully predicting a seizure before it begins. However, epilepsy prediction remains one of the competitive challenges for researchers, and numerous methods have already been proposed to address this problem. Scholars approach this issue in various ways, e.g., linear methods (Salant et al., 1998) and nonlinear dynamics (Iasemidis et al., 1990). For linear measurement, statistical measures, including the calculation of variance, skewness, and kurtosis, are used for several seizure prediction tools, and researchers have described that kurtosis increase but variance decrease during the state of preictal activity (Aarabi et al., 2009). The mean phase coherence (MPC) (Mormann et al., 2003), Shannon entropy index (Rosenblum et al., 2000), and conditional probability index are the best nonlinear measures compared to other nonlinear features (Mormann et al., 2005). Moreover, the differential entropy with the cumulative sum (CUSUM) procedure has been applied to predict seizures and shows $87.5 \%$ sensitivity, a 0.28 per hour false prediction rate and a 25 minute average prediction time (Zandi et al., 2009). In other studies, the permutation entropy (PE) method has been used to extract features, and combined with a support vector machine (SVM) classification method, $94 \%$ sensitivity, a 0.111 per hour false prediction rate, and 63.93 minutes of the average prediction time were shown (Yang et al., 2018).

There are different types of methods for measuring time series complexity, e.g., entropies (Coifman \& Wickerhauser, 1992), fractal dimensions (Mashiah et al., 2008), and Lyapunov exponents (Rosenstein et al., 1993). However, entropy calculation becomes more interesting in the neuroscience field because of the nonstationary features of the EEG signals. Entropy is a method that can be used to distinguish the regular, chaotic, and random behavior of a time series by measuring complexity (Paluš, 1998). Moreover, the use of entropy combined with a Monte Carlo tree search (MCTS) process is the most effective method to addredd the container loading problem (Cant et al., 2018); therefore, entropy is a method that can be used to measure disorder or irregularities in a wide range of applications (Howedi et al., 2020). Additionally, EEG signals 
79 from epileptic patients can be classified into three different states: interictal state, preictal state,

80 and ictal state (see Fig. 1). The first state refers to the time between seizures, the second state is 81 the time period just before the seizure arrives, and the last state is the seizure period (Chiang et

82 al., 2011). In previous work, a new entropy method called modified-distribution entropy

83 (mDistEn) was proposed, and this method successfully detects the different states of epileptic

84 EEG signals by calculating the complexity of the signals (Aung \& Wongsawat, 2020). Moreover,

85 an effective coarse-grained calculation was added to the entropy method, which becomes

86 multiscale modified-distribution entropy (M-mDistEn) (Aung \& Wongsawat, 2021). The

87 purpose of this multiscale method is to detect the various scales (frequencies) of the EEG

88 signals; therefore, this method is usable for detecting motion artifacts. The main difference

89 between the common entropy and the multiscale entropy is the evaluation of time series coarse-

90 grained entropy to quantify the interdependency between entropy and scales (Costa et al., 2002).

91 However, there are some limitations to multiscale entropy because it is designed for scalar time

92 series analysis, and it is not suitable for accurately reflecting the complexity of multivariate time

93 series in complex systems (Zhang \& Shang, 2019).

94 The advantages of multivariate entropy can overcome the shortcomings of multiscale entropy,

95 including evaluating within- and cross-channel dependencies in multiple data channels, assessing

96 of the underlying dynamical richness of multichannel observations, and more degrees of freedom

97 in the analysis than those of standard multiscale entropy (Ahmed \& Mandic, 2011). For the

98 reasons outlined above, entropy can distinguish the different states of epileptic EEG signals, and

99 therefore, the prediction of epilepsy is possible according to numerous experiments (Yang et al.,

100 2018). By exploring the advantages of multivariate methods and the previous entropy methods

101 (mDistEn and M-mDistEn), a new method called MM-mDistEn is proposed, and this new

102 method provides the crucial features extracted from epileptic EEG signals and applies these

103 features to ANNs (Siddique \& Adeli, 2013) in seizure prediction systems. The proposed system

104 also reveals improved results in all performance measures; thus, it may be another alternative

105 method for helping epileptic patients predict seizures before they start.

106

107 Materials \& Methods

108 A detailed explanation of the calculation of the proposed entropy method, MM-mDistEn, is

109 mentioned in this section. The classification of epilepsy is also performed by using an ANN, and

110 the step-by-step procedure is described after the explanation of the parameter selection. In this

111 paper, the public dataset is used for experimental data analysis and introduced in the next

112 subsection.

113

114 Dataset description

115 The signals used for analysis in this paper are from the public PhysioNet Database (Goldberger

116 et al., 2000; Shoeb, 2009), where the CHB-MIT dataset of EEG signals with seizure events is

117 used for data analysis. The dataset is freely downloadable via the link that is provided in the

118 reference section (CHB-MIT, 2009). Data were collected as previously described in (Daoud \&

PeerJ Comput. Sci. reviewing PDF | (CS-2021:07:63740:1:1:NEW 2 Sep 2021) 
119 Bayoumi, 2019) and which include long-term scalp EEG data form pediatric subjects with

120

121

122

123

124

125

126

127

128

129

130

131

132

133

134

135

136

137

138

139

140

141

142

143

144

145

146

147

148

149

150

151

152

intractable seizures. Recordings, grouped into 23 cases, were collected from 22 subjects ( 5 males, ages 3-22; and 17 females, ages 1.5-19), and these subjects were monitored for up to several days following withdrawal of antiseizure medication to characterize their seizures and evaluate their candidacy for surgical intervention (Shoeb, 2009). Most files contain 23 EEG signals ( 24 or 26 in a few cases). The international 10-20 system of EEG electrode positions and the sampling rate is 256 per second with 16-bit resolution (Goldberger et al., 2000). Fig. 1 shows a one-hour recording of epileptic EEG signals from subject no.1. The different states of interictal, preictal, and ictal of EEG signals from the frontal area, occipital area, and different area of the brain are clearly shown in Fig. 1.

\section{Multivariate multiscale modified-distribution entropy}

A new method, MM-mDistEn, was used to calculate the data multidimensionally. Three steps are required to calculate the entropy values, as shown in Fig. 2.

The algorithm is calculated as follows:

Step 1. Multivariate time series

First, the multivariate time series from the given time series data is constructed. The new input multichannel EEG can be set and notated as $x_{c, i}$ where $c$ is the number of channels $(1,2$, $\ldots, C)$, and $i$ is the number of samples in each channel $(1,2, \ldots, N)$.

Step 2. Coarse-graining process

The coarse-grained time series can be calculated using the multivariate time series data according to the scale factor, and the equation can be expressed as

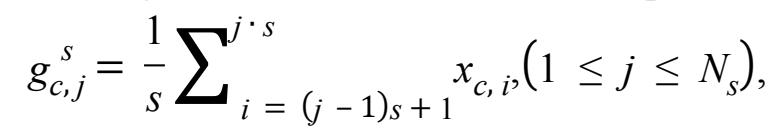

where $g_{c, j}^{s}$ is the multivariate coarse-grained time series, $s$ is a scale factor and $N_{s}=\frac{N}{s}$.

Step 3. Calculate MM-mDistEn

Phase-space reconstruction is performed before the calculation of the entropy values, and the reconstruction is as follows:

$\mathbf{M}^{s}(j)=\left[\begin{array}{cccc}g_{1, j}^{s} & g_{1, j+\tau} & \cdots & g_{1, j+(m-1) T} \\ g_{2, j} & g_{2, j+\tau} & \cdots & g_{2, j+(m-1) \tau} \\ \vdots & \vdots & \vdots & \vdots \\ g_{c, j}^{s} & g_{c, j+\tau} & \cdots & g_{C, j+(m-1) T}\end{array}\right],\left(1 \leq j \leq N_{s}\right)$,

where $m$ is the embedding dimension and $\tau$ is the time delay. For the current study, $m=3$ and $\tau=1$ are used (more information available in the parameter selection subsection below).

The proposed method, MM-mDistEn, which is implemented based on distribution entropy, adds two more threshold parameters, ' $r$ ' and ' $n$ ', to existing parameters. $r$ is set by multiplying the standard deviation of all data values by 0.2 , and $n$ is set to 2 (Aung \& Wongsawat, 2020). For a given multivariate coarse-grained time series, 
153

154

155

156

157

(i) Create matrix $\mathbf{X}^{s}(j)$ in term of $\mathbf{M}^{s}(j)$ by

$\mathbf{X}^{s}(j)=\left[\mathbf{M}^{s}(j), \mathbf{M}^{s}(j+1), \ldots, \mathbf{M}^{s}(j+(m-1) \tau)\right],\left(1 \leq j \leq N_{s}-(m-1)\right)$.

(ii) The distance matrix $\mathbf{D}_{i j}^{s}$ is computed as $\hat{\mathbf{D}}_{i j}^{s}$ divided by $r$ and then squared $(n=2)$ :

$\mathbf{D}_{i j}^{s}=\left(\frac{\hat{\mathbf{D}}_{i j}^{s}}{r}\right)^{n}$.

158

159

160

(iii) After obtaining $\mathbf{D}_{i j}^{S}$, the empirical probability density function (ePDF) is calculated using the histogram approach from $\mathbf{D}_{i j}^{S}$ of the previous steps with the bin number, $B$. The probability for that number can be given as $\mathrm{P}_{t}$, where $t=1,2,3, \ldots, B$.

163

164

165

166

167

168

169

A matrix $\hat{\mathbf{D}}_{i j}^{s}$ between $\mathbf{X}^{s}(i)$ and $\mathbf{X}^{s}(j)\left(1 \leq i, j \leq N_{s}-(m-1), i \neq j\right)$ is computed using the Euclidean method.

170

(iv) MM-mDistEn can be calculated by the following equation with the distance matrix $\mathbf{D}_{i j}^{S}$ as follows:

$\operatorname{MM-mDistEn}(m, \tau, r, n, B, s)=-\frac{1}{\log _{2}(B)} \sum_{t=1}^{B} \mathrm{P}_{t}\left(\mathbf{D}_{i j}^{s}\right) \log _{2}\left[\mathrm{P}_{t}\left(\mathbf{D}_{i j}^{s}\right)\right]$,

171

172

173

174

175

176

177

178

179

180

181

182

183

184

185

186

187

$$
\left(1 \leq i, j \leq N_{s}-(m-1), i \neq j\right) \text {. }
$$

\section{Parameter selection}

MM-mDistEn uses predefined values for selecting parameters. There are six parameters that are required to compute the entropy values. First, the time delay, $\tau$ and dimension, $m$ are used for the reconstructing of phase-space, with values of 1 and 3, respectively ( $\mathrm{Li}$ et al., 2015b). Next, the distance matrix, $\mathrm{D}_{\mathrm{ij}}^{\mathrm{s}}$, is calculated using the parameters $r$ and $n$, where $r$ is the tolerance number and $n$ is the order of the function, and both parameters are applied in calculating the proposed entropy. A large $r$ and $n$ can lead to noise influence, whereas a small $r$ and $n$ can cause information lose; and therefore, the parameter $r$ is equal to the standard deviation of the series multiplied by 0.2 , and $n$ is set to 2 (Aung \& Wongsawat, 2020; Chen et al., 2007). When the ePDF is calculated, another parameter value called the bin number, $B$, is needed, and $B$ is set to 64 for this estimation ( $\mathrm{Li}$ et al., 2016). The scale factor, $s$, is also needed for calculating of the multivariate multiscale entropy values, and the scale values used in data analysis range from 1 to 15 (Acharya et al., 2015). Additionally, the different values are selected for these parameters and shown in the figures in results section.

\section{Classification of epileptic seizures from the extracted features}

In this paper, a multilayer perceptron (MLP), which is an ANN, is used for training and testing the data. First, feature calculation is performed by using the MM-mDistEn method before these features are input to the ANN. After that, the neural networks are implemented by using the library support from TensorFlow. These features are imported into the environment for 
188

189

190

191

192

193

194

195

196

197

198

199

200

201

202

203

204

205

206

207

208

209

210

211

212

213

214

215

216

217

218

219

220

221

222

223

224

225

226

227

calculation and separated into input data and target data, and then these data are split into two sets: preictal period for the test set and interictal period for training. The 12 units for the first hidden layer and second hidden layer are used in the neural networks. The model was trained with backpropagation and optimized with the RMSprop algorithm (Daoud \& Bayoumi, 2019). The loss function used in this model is the binary cross entropy (Daoud \& Bayoumi, 2019). The rectified linear unit (ReLU) activation function (Hahnloser et al., 2000) is used for the hidden layers to add nonlinearity and make strong robustness to clear the noise from the input data. The softmax activation function is selected for the output layer to classify the multiclass outputs, and interictal, preictal, and ictal states of the epileptic EEG signals (Usman et al., 2020). The networks for each patient are trained individually for all 24 subjects. Finally, the results are shown in the results section.

\section{Seizure alarm system}

The calculation of mean values is performed when the predicted features $\left(\mathrm{P}_{\mathrm{F}}\right)$ are generated from the ANN, and those values are used as the decision-making process of the seizure alarm system. The seizure features $\left(\mathrm{S}_{\mathrm{F}}\right)$ are selected from the duration of the ictal period of the EEG signals, and the mean values of those periods are calculated. Then, the mean values of $\mathrm{S}_{\mathrm{F}}$ and $\mathrm{P}_{\mathrm{F}}$ are used for comparison, i.e., if the former values are greater than or equal to the latter values, the alarm signal is triggered for upcoming seizure events. The flow chart for the seizure alarm system is shown in Fig. 3.

\section{Results}

In this section, two different analyses are conducted with two different datasets. The simulation data are used to test the consistency of the proposed entropy by changing the different parameters values. The experimental dataset is required for the next process of classification and epilepsy prediction. A detailed explanation of the results from these two analyses is described in the following subsections.

\section{Analysis of simulation signals}

The proposed entropy method is analyzed with two different datasets: simulation data and experimental data. For the first dataset, three different signal types; sine wave (50 Hz frequency), chaotic series, and Gaussian series are used (Li et al., 2015a), and the length of each signal is 400 samples. After that, these three series are simulated as a function of the scale factor with embedding dimensions and shown in Fig. 4. In Fig. 4 (a) and (c), these three series are plotted according to their entropy values, but the chaotic series and Gaussian series overlap with each other in embedding parameters of values 2 and 4 . However, the entropy values of three different series plot well-defined over all scale factors (1 to 15) with an embedding parameter value of $m=3$ and are visualized in Fig. 4 (b). Therefore, the value of 3 for the embedding parameter is chosen, and the detailed explanation of the parameters used in the calculation of MM-mDistEn is described in the parameter selection section. Although the dependence of MM-mDistEn is on the bin

PeerJ Comput. Sci. reviewing PDF | (CS-2021:07:63740:1:1:NEW 2 Sep 2021) 
228

229

230

231

232

233

234

235

236

237

238

239

240

241

242

243

244

245

246

247

248

249

250

251

252

253

254

255

256

257

258

259

260

261

262

$263 \mathrm{R}_{\mathrm{fa}}=\frac{\mathrm{N}_{\mathrm{fa}}}{\mathrm{H}_{\mathrm{t}}}$, number, all three different series have upward trends regarding the bin values, $B\left(2^{0}\right.$ to $\left.2^{9}\right)$, and distinguish these three series, as shown in Fig. 5 (a). The time delay, $\tau$ values range from 1 to 10, and the MM-mDistEn values decrease with increasing parameter values (see Fig. 5 (b)). In Fig. 5 (c), the tolerance numbers, $r$ used in the entropy method are multiplied by the standard deviation of the series, and these values range from 0.05 to 0.9 with an increase of 0.05 . The plotted MMmDistEn values are chaotic, Gaussian and sine waves on the order of higher to lower values.

\section{Analysis of the experimental data}

The values of MM-mDistEn are plotted with the scale factors (1 to 15) and are shown in Fig. 6. Instead of plotting all datasets, the entropy values of epileptic EEG signals from four subjects are plotted, and the entropy values are different in order of the highest values for the ictal state and the lowest values for the preictal state (see Fig. 6 (a), (c), and (d)). The highest complexity is for ictal EEG signals, which are deterministic chaotic dynamics, compared with normal EEG signals, which are stochastic dynamics (Li et al., 2015b). Although a different order is seen in Fig. 6 (b), MM-mDistEn can still distinguish the three states of the epileptic EEG signals. The performance measure is performed by calculating the accuracy, sensitivity, specificity, and AUC (Li et al., 2018) for all the subjects from the CHB-MIT dataset.

$$
\text { Accuracy }=\frac{\mathrm{TP}+\mathrm{TN}}{\mathrm{TP}+\mathrm{FP}+\mathrm{TN}+\mathrm{FN}} \text {, }
$$

Sensitivity $=\frac{\mathrm{TP}}{\mathrm{TP}+\mathrm{FN}}$

$$
\text { Specificity }=\frac{\mathrm{TN}}{\mathrm{TN}+\mathrm{FP}},
$$

where TP and TN are the number of true positives and the number of true negatives, i.e., the classifier correctly labels the actual number of ictal and normal EEG signals and FP and FN are the number of false positive and false negatives, these two values indicate the number of ictal and normal signals that are incorrectly categorized by the classifier ( $\mathrm{Li}$ et al., 2018). It is clearly shown that the proposed extraction method combined with an ANN achieves an average performance measure of 98.66\% accuracy, 91.82\% sensitivity, 99.11\% specificity, and an AUC value of 0.84 (see Table 1). Moreover, the minimum scores of the performance measure are still effective with an accuracy of $95.2 \%$, a sensitivity of $83 \%$, a specificity of $93.67 \%$, and an AUC value of 0.75 (among all 24 subjects, in Fig. 7).

\section{Performance measures for predicting epileptic EEG signals}

The performance of the proposed prediction algorithm is calculated based on three factors: the false alarm rate $\left(R_{f a}\right)$, the prediction time, and the prediction rate $\left(R_{p}\right)$ (Aarabi \& He, 2014):

$$
R_{f a}=\frac{N_{f a}}{H_{t}}
$$


264

$265 R_{p}=\frac{N_{p}}{N_{t}}$

$266 \mathrm{~T}_{\text {avg }}=\frac{\mathrm{T}_{\mathrm{p}}}{\mathrm{N}_{\mathrm{p}}}$,

267 where $\mathrm{N}_{f a}$ is the number of false alarms, $\mathrm{H}_{t}$ is the total number of hours, $\mathrm{N}_{\mathrm{p}}$ is the number of predicted seizures, $N_{t}$ is the total number of seizures, $T_{\text {avg }}$ is the average prediction time and $T_{p}$ is the prediction time. The performance values of all cases of the 24 subjects are shown in Fig. 8 .

270 The prediction time is defined as the time between the instant at which a seizure can be

271 predicted, and the actual beginning of the seizure and 1-hour (3600 seconds) long epileptic EEG

272 signals are used for the prediction system (see Fig. 9). A $\mathrm{T}_{\text {avg }}$ of 26.73 minutes is achieved among all cases from the 24 subjects. The proposed method with an ANN achieves an average

274 false alarm rate of under 0.25 per hour, an average prediction rate of over $70 \%$, and an average

275 training time of fewer than 3.5 minutes (see Fig. 8).

276

\section{Discussion}

278 According to previous works (Aung \& Wongsawat, 2020), there are some limitations on

279 multivariate time series analysis, and therefore, MM-mDistEn is proposed to overcome these

280

281

282

283

284

285

286

287

288

289

290

291

292

293

294

295

296

297

298

299

300

301 limitations. First, the phase-space reconstruction and estimation of the probability density between vectors provide hidden complex information. The multivariate time series property of MM-mDistEn gives us more understandable information within the multichannel data. Moreover, the data are also analyzed at different scales (frequencies) so that insight information can be seen in different scales ranges. According to the above results section, the proposed method was tested with two different analyses: simulation data and experimental data. Testing with the simulation data is used if the information of the proposed entropy has strong consistency and less dependency on preset parameters. Regarding the experimental data, the different performance measures are also provided on the proposed entropy combined with an ANN to classify the three states of the seizure from epileptic EEG signals. Additionally, postprocessing of the seizure alarm system helps patients predict upcoming seizures before they occur. The different performance measures on the proposed MM-mDistEn are illustrated in Fig. 7, and the performance evaluation for the prediction of epileptic EEG signals is described in Fig. 8. A summary of the performance comparison between the existing prediction methods that have used the same dataset and the proposed method is shown in Table 1. The proposed method obtains a higher accuracy score among these methods, excluding the method using a convolutional neural network (CNN) (Gómez et al., 2020), while the training time is shorter than that of the other methods such as M-mDistEn with an ANN, PE with an ANN, a deep convolutional neural network (DCNN) with MLP and MLP (Daoud \& Bayoumi, 2019). The sensitivity of the proposed method obtains a better score than other methods, but it is slightly lower than DCNN with MLP and CNN with SVM. Although the specificity of the proposed entropy is marginally lower than that of the method using $\mathrm{CNN}$, the best rate for false alarms is

PeerJ Comput. Sci. reviewing PDF | (CS-2021:07:63740:1:1:NEW 2 Sep 2021) 
302 obtained by the proposed method. The false alarm rate is also crucial for the prediction of 303 epilepsy, and it is the smallest rate among these methods. An important factor in the prediction of 304 epilepsy is the prediction time because it enables the delivery of warning signals to patients in a 305 timely manner. The proposed combined system of MM-mDistEn and an ANN can send an alarm 306 on average 26.73 minutes before the actual seizure starts according to the results from the 307 experiments in all 24 subjects; therefore, the prediction time of the proposed method is earlier 308 than that with the method using a CNN with SVM (Usman et al., 2020).

309

\section{Conclusions}

311 In this paper, a new feature extraction method, called MM-mDistEn, was proposed for predicting 312 of seizures through combination with an ANN. The proposed method efficiently explores the 313 information from multiple variables with multiple time scales and analyzes the complexity of 314 that time series. Two different analyses were performed: a simulation dataset is used to prove the 315 existence of consistency, and an experimental dataset is applied to distinguish of the different 316 states of epileptic EEG signals. The performance measures of the proposed method were 317 provided for the classification of the interictal, preictal, and ictal states. The advantages of 318 multivariate robust entropy provide an efficient method for extracting features from multichannel 319 EEG recordings. Moreover, the seizure alarm system was added as postprocessing step, which 320 can warn patients about an oncoming seizure before its onset by providing an adequate 321 prediction of the time between the preictal and ictal states. The proposed combination method 322 will only require an EEG acquisition system for real-time usage, and it can become useful not 323 only for clinical applications but also for usage outside of the hospital for epilepsy patients. 324 Therefore, a portable version for seizure prediction can become a reality by using the proposed 325 method. Future studies are needed for real-time applications to detect more complex behaviors 326 from the different EEG datasets.

327

\section{Acknowledgements}

329 This project is supported by the National Higher Education Science Research and Innovation 330 Policy Council, PMU B. There was no additional external funding received for this study. 
331

332

333

334

335

336

337

338

339

340

341

342

343

344

345

346

347

348

349

350

351

352

353

354

355

356

357

358

359

360

361

362

363

364

365

366

367

368

369

370

371

372

373

374

\section{References}

Aarabi A, Fazel-Rezai R, and Aghakhani Y. 2009. EEG seizure prediction: measures and challenges. 2009 Annual International Conference of the IEEE Engineering in Medicine and Biology Society: IEEE. p 1864-1867.

Aarabi A, and He B. 2014. Seizure prediction in hippocampal and neocortical epilepsy using a model-based approach. Clinical neurophysiology 125:930-940.

Acharya UR, Fujita H, Sudarshan VK, Bhat S, and Koh JE. 2015. Application of entropies for automated diagnosis of epilepsy using EEG signals: A review. Knowledge-Based Systems 88:85-96.

Ahmed MU, and Mandic DP. 2011. Multivariate multiscale entropy: A tool for complexity analysis of multichannel data. Physical Review E 84:061918.

Aung ST, and Wongsawat Y. 2020. Modified-distribution entropy as the features for the detection of epileptic seizures. Frontiers in Physiology 11:607.

Aung ST, and Wongsawat Y. 2021. Analysis of EEG Signals Contaminated With Motion Artifacts Using Multiscale Modified-Distribution Entropy. IEEE Access 9:33911-33921.

Cant R, Remi-Omosowon A, Langensiepen C, and Lotfi A. 2018. An Entropy-Guided Monte Carlo Tree Search Approach for Generating Optimal Container Loading Layouts. Entropy 20:866.

CHB-MIT scalp EEG database. 2009. Available at https://www.physionet.org/content/chbmit/1.0.0/ (accessed 2 May 2020)

Chen W, Wang Z, Xie H, and Yu W. 2007. Characterization of surface EMG signal based on fuzzy entropy. IEEE Transactions on neural systems and rehabilitation engineering 15:266-272.

Chiang C-Y, Chang N-F, Chen T-C, Chen H-H, and Chen L-G. 2011. Seizure prediction based on classification of EEG synchronization patterns with on-line retraining and postprocessing scheme. 2011 Annual International Conference of the IEEE Engineering in Medicine and Biology Society: IEEE. p 7564-7569.

Coifman RR, and Wickerhauser MV. 1992. Entropy-based algorithms for best basis selection. IEEE Transactions on information theory 38:713-718.

Costa M, Goldberger AL, and Peng C-K. 2002. Multiscale entropy analysis of complex physiologic time series. Physical review letters 89:068102.

Daoud H, and Bayoumi MA. 2019. Efficient epileptic seizure prediction based on deep learning. IEEE transactions on biomedical circuits and systems 13:804-813.

Fujiwara K, Miyajima M, Yamakawa T, Abe E, Suzuki Y, Sawada Y, Kano M, Maehara T, Ohta K, and Sasai-Sakuma T. 2015. Epileptic seizure prediction based on multivariate statistical process control of heart rate variability features. IEEE Transactions on Biomedical Engineering 63:1321-1332.

Goldberger AL, Amaral LA, Glass L, Hausdorff JM, Ivanov PC, Mark RG, Mietus JE, Moody GB, Peng C-K, and Stanley HE. 2000. PhysioBank, PhysioToolkit, and PhysioNet: components of a new research resource for complex physiologic signals. circulation 101:e215-e220.

Gómez C, Arbeláez P, Navarrete M, Alvarado-Rojas C, Le van Quyen M, and Valderrama M. 2020. Automatic seizure detection based on imaged-EEG signals through fully convolutional networks. Scientific reports 10:1-13. 
375

376

377

378

379

380

381

382

383

384

385

386

387

388

389

390

391

392

393

394

395

396

397

398

399

400

401

402

403

404

405

406

407

408

409

410

411

412

413

414

415

416

417

418

419

Hahnloser RH, Sarpeshkar R, Mahowald MA, Douglas RJ, and Seung HS. 2000. Digital selection and analogue amplification coexist in a cortex-inspired silicon circuit. Nature 405:947-951.

Howedi A, Lotfi A, and Pourabdollah A. 2020. An entropy-based approach for anomaly detection in activities of daily living in the presence of a visitor. Entropy 22:845.

Iasemidis LD, Sackellares JC, Zaveri HP, and Williams WJ. 1990. Phase space topography and the Lyapunov exponent of electrocorticograms in partial seizures. Brain topography 2:187-201.

Li P, Karmakar C, Yan C, Palaniswami M, and Liu C. 2016. Classification of 5-S epileptic EEG recordings using distribution entropy and sample entropy. Frontiers in Physiology 7:136.

Li P, Karmakar C, Yearwood J, Venkatesh S, Palaniswami M, and Liu C. 2018. Detection of epileptic seizure based on entropy analysis of short-term EEG. PloS one 13:e0193691.

Li P, Liu C, Li K, Zheng D, Liu C, and Hou Y. 2015a. Assessing the complexity of short-term heartbeat interval series by distribution entropy. Medical \& biological engineering \& computing 53:77-87.

Li P, Yan C, Karmakar C, and Liu C. 2015b. Distribution entropy analysis of epileptic EEG signals. 2015 37th Annual International Conference of the IEEE Engineering in Medicine and Biology Society (EMBC): IEEE. p 4170-4173.

Mashiah A, Wolach O, Sandbank J, Uziel O, Raanani P, and Lahav M. 2008. Lymphoma and leukemia cells possess fractal dimensions that correlate with their biological features. Acta haematologica 119:142-150.

Mormann F, Kreuz T, Andrzejak RG, David P, Lehnertz K, and Elger CE. 2003. Epileptic seizures are preceded by a decrease in synchronization. Epilepsy research 53:173-185.

Mormann F, Kreuz T, Rieke C, Andrzejak RG, Kraskov A, David P, Elger CE, and Lehnertz K. 2005. On the predictability of epileptic seizures. Clinical neurophysiology 116:569-587.

Paluš M. 1998. Chaotic measures and real-world systems. Nonlinear analysis of physiological data: Springer, 49-66.

Rosenblum M, Tass P, Kurths J, Volkmann J, Schnitzler A, and FREUND H-J. 2000. Detection of phase locking from noisy data: application to magnetoencephalography. Chaos In Brain?: World Scientific, 34-51.

Rosenstein MT, Collins JJ, and De Luca CJ. 1993. A practical method for calculating largest Lyapunov exponents from small data sets. Physica D: Nonlinear Phenomena 65:117-134.

Salant Y, Gath I, and Henriksen O. 1998. Prediction of epileptic seizures from two-channel EEG. Medical and Biological Engineering and Computing 36:549-556.

Shoeb AH. 2009. Application of machine learning to epileptic seizure onset detection and treatment. Massachusetts Institute of Technology.

Siddique N, and Adeli H. 2013. Computational intelligence: synergies of fuzzy logic, neural networks and evolutionary computing: John Wiley \& Sons.

Usman SM, Khalid S, and Aslam MH. 2020. Epileptic seizures prediction using deep learning techniques. IEEE Access 8:39998-40007.

Wang C, Zou J, Zhang J, Wang M, and Wang R. 2010. Feature extraction and recognition of epileptiform activity in EEG by combining PCA with ApEn. Cognitive neurodynamics 4:233-240.

World Health Organization (WHO). 2019, Epilepsy. Available at https://www.who.int/newsroom/fact-sheets/detail/epilepsy (accessed 9 May 2021)

Peer) Comput. Sci. reviewing PDF | (CS-2021:07:63740:1:1:NEW 2 Sep 2021) 
420 Yang Y, Zhou M, Niu Y, Li C, Cao R, Wang B, Yan P, Ma Y, and Xiang J. 2018. Epileptic

421

422

423

424

425

426

427 seizure prediction based on permutation entropy. Frontiers in computational neuroscience 12:55.

Zandi AS, Dumont GA, Javidan M, and Tafreshi R. 2009. An entropy-based approach to predict seizures in temporal lobe epilepsy using scalp EEG. 2009 Annual International Conference of the IEEE Engineering in Medicine and Biology Society: IEEE. p 228-231.

Zhang Y, and Shang P. 2019. Multivariate multiscale distribution entropy of financial time series. Physica A: Statistical Mechanics and its Applications 515:72-80. 


\section{Figure 1}

Epileptic EEG signals from subject no.1 (a) frontal area, (b) occipital area, and (c) different area including central, temporal, and parietal brain areas.

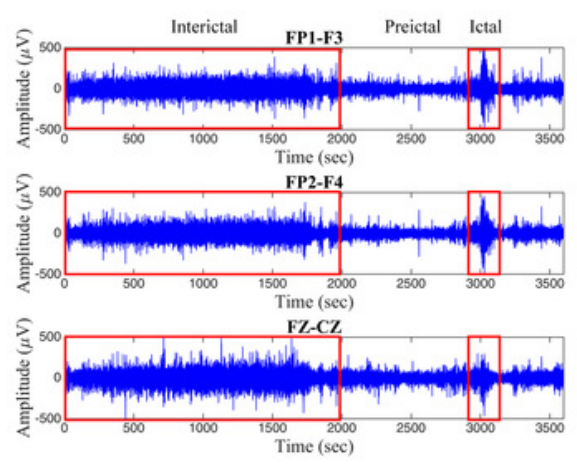

(a)

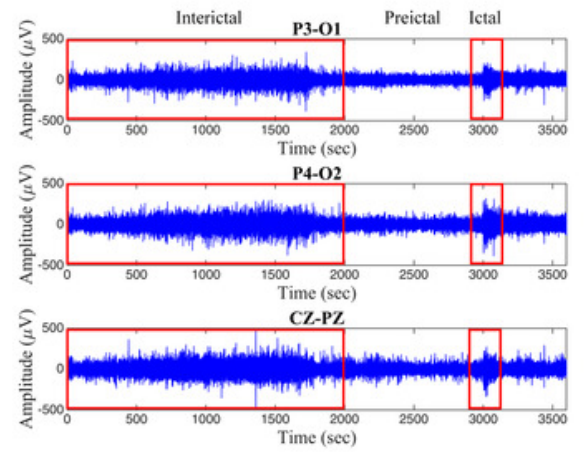

(b)

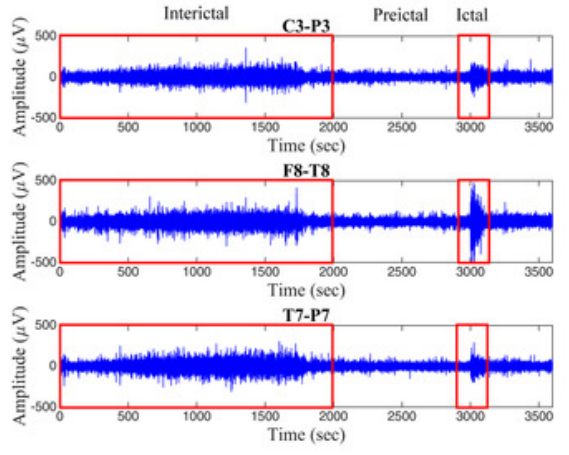

(c) 
Figure 2

Block diagram for calculating multivariate multiscale modified-distribution entropy.

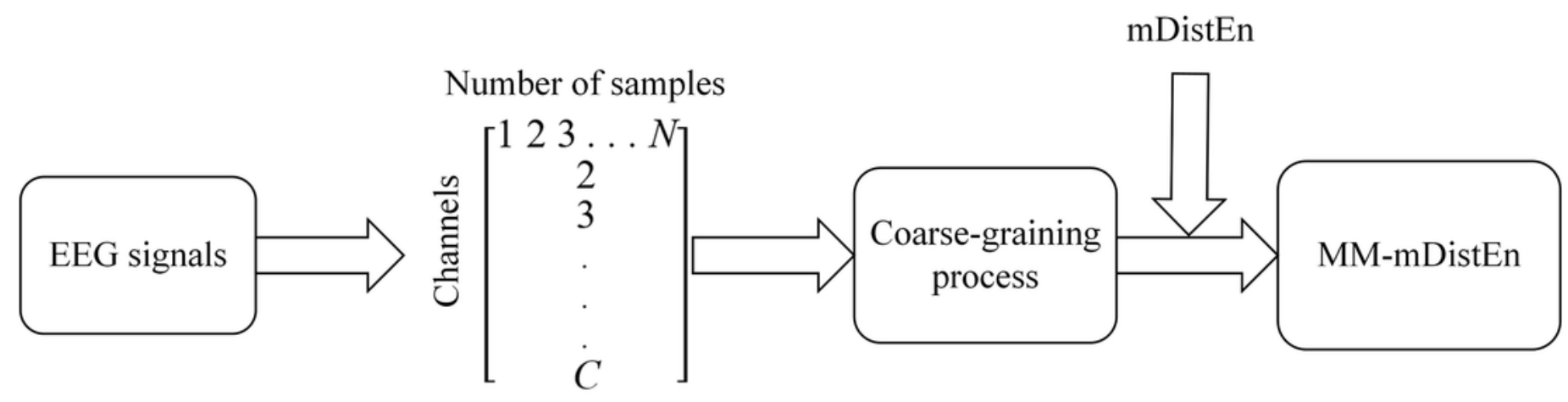

Multivariate time series 
Figure 3

Flow chart algorithm for the seizure alarm system.

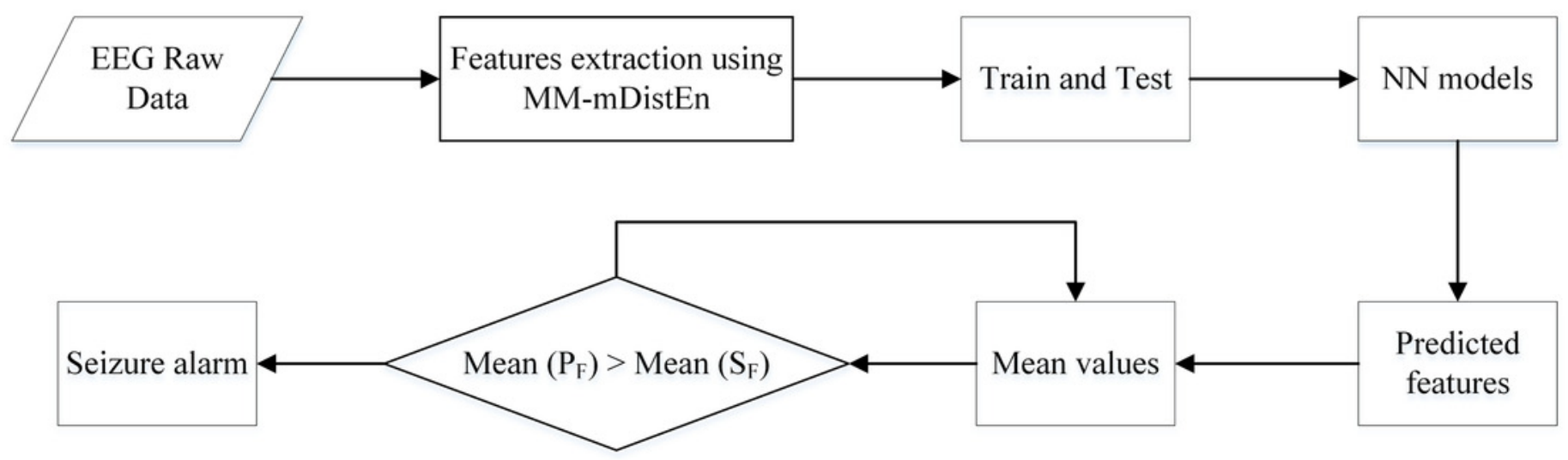


Figure 4

The values of entropies (unitless) on simulation data as a function of the scale factor with different embedding dimensions (a) $m=2$, (b) $m=3$, and (c) $m=4$.

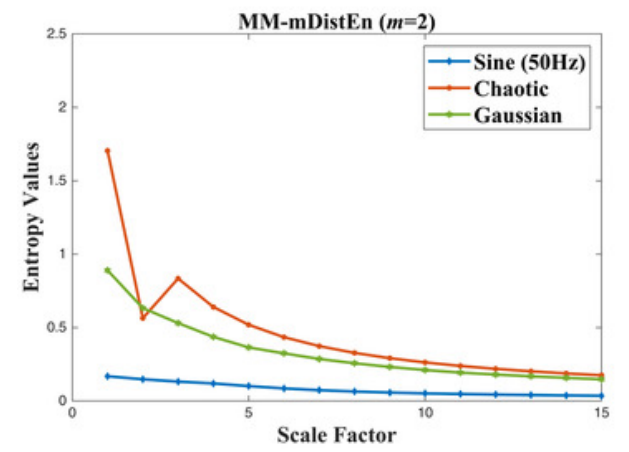

(a)

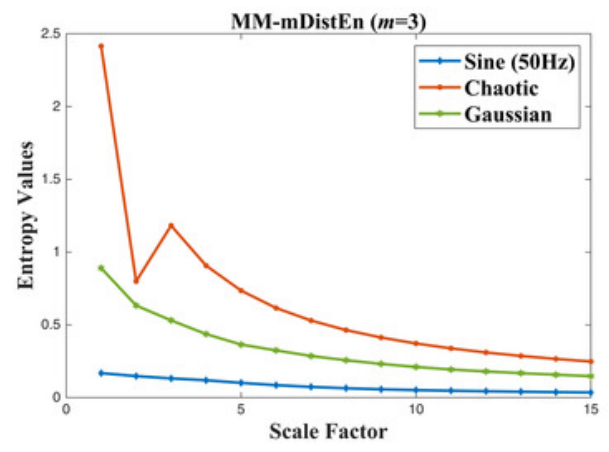

(b)

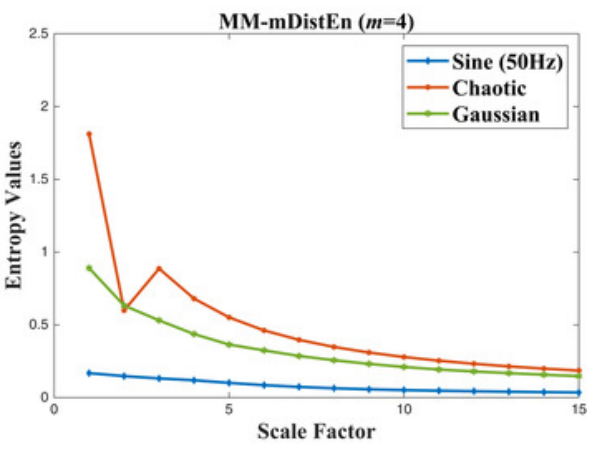

(c) 
Figure 5

The entropy values (unitless) on simulation data with respect to the: (a) number of bins $\left(2^{0}\right.$ to $\left.2^{9}\right)$, (b) time delay ( 1 to 10$)$, and (c) tolerance ( 0.05 to 0.9 multiplied by the standard deviation of the simulation data).

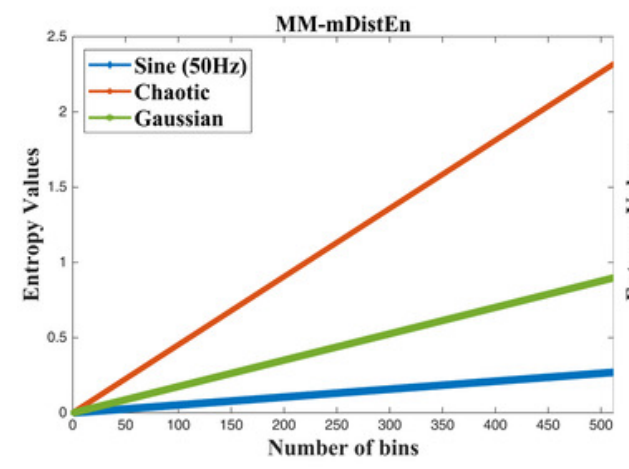

(a)

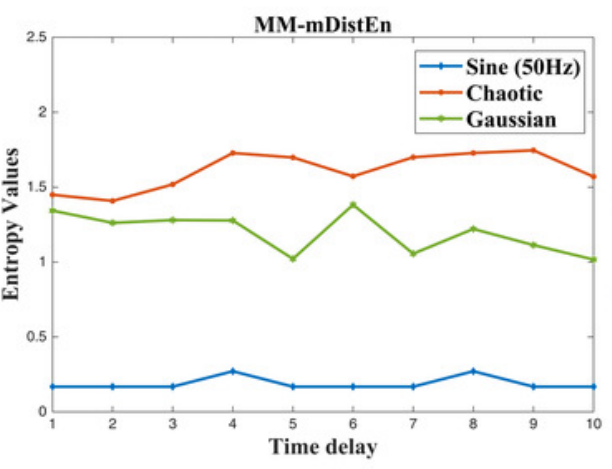

(b)

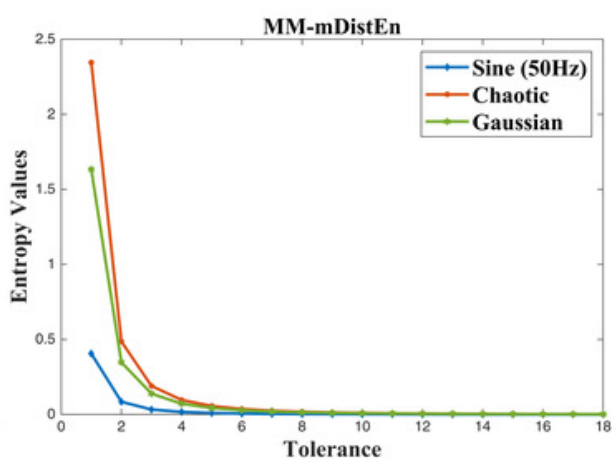

(c) 
Figure 6

The values of MM-mDistEn (unitless) in the experimental dataset with different subjects (a) subject no.1, (b) subject no.4, (c) subject no.7, and (d) subject no.8.

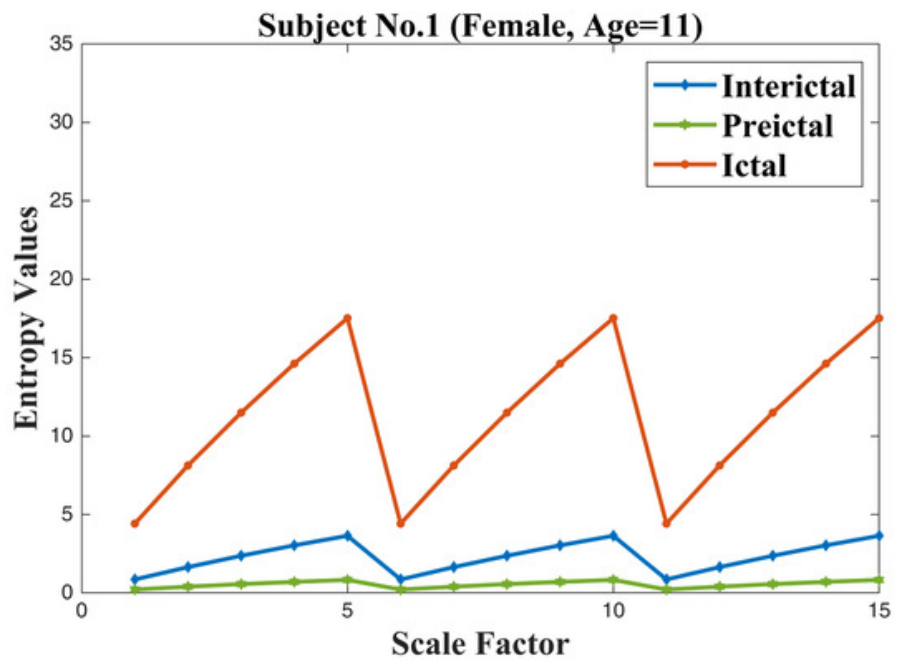

(a)

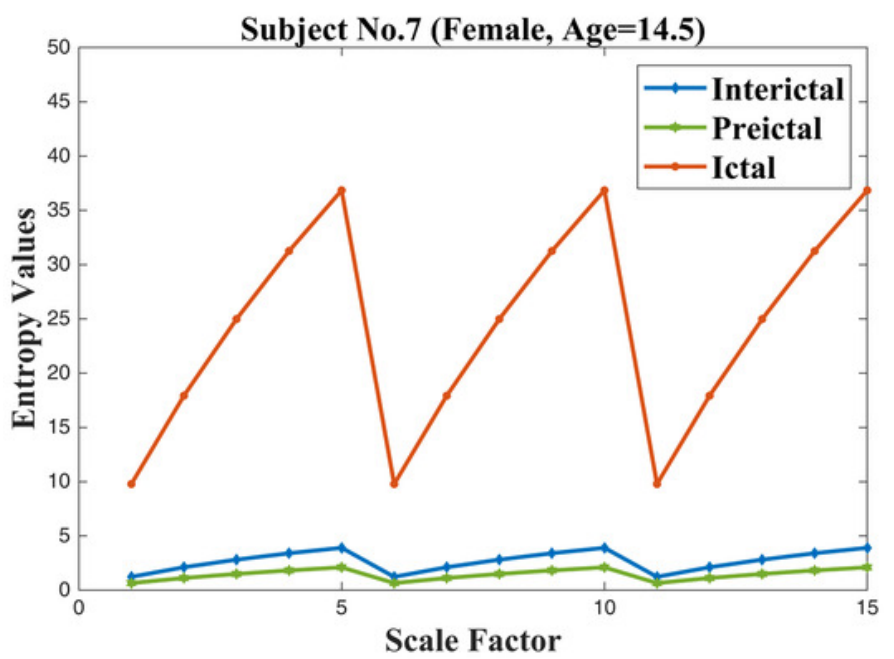

(c)

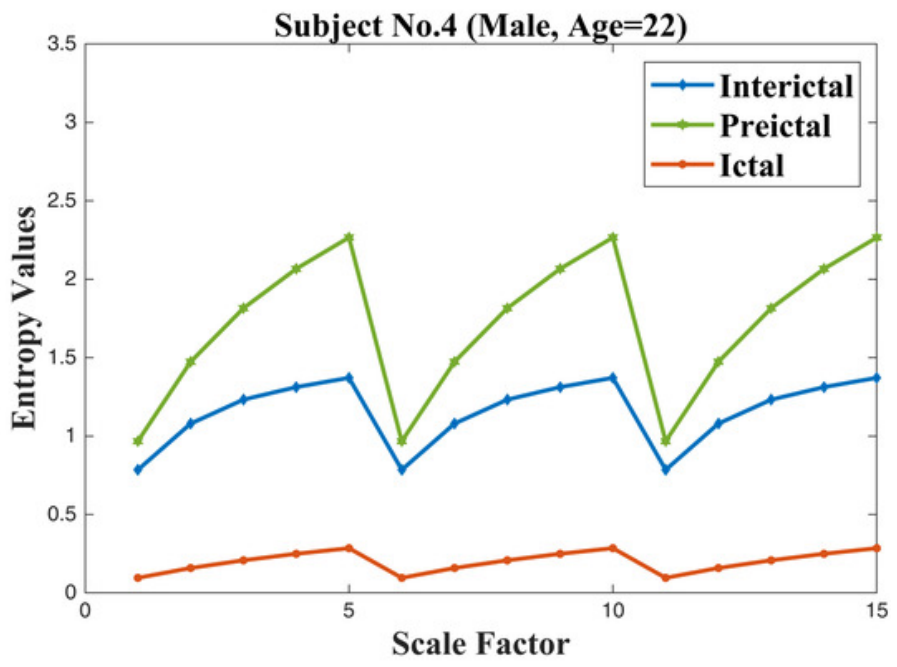

(b)

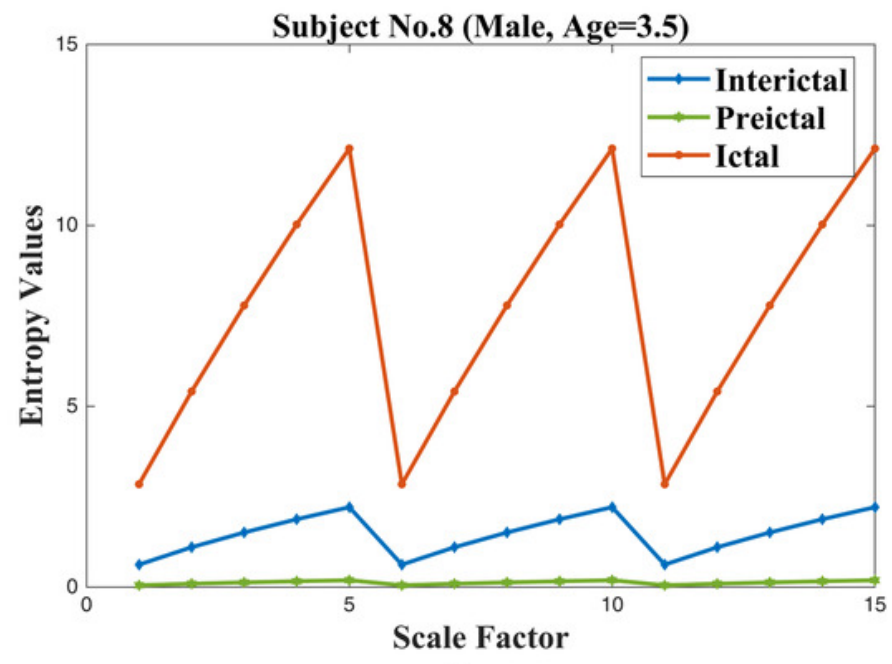

(d) 
Figure 7

Performance measures for the analysis of EEG signals with an ANN.
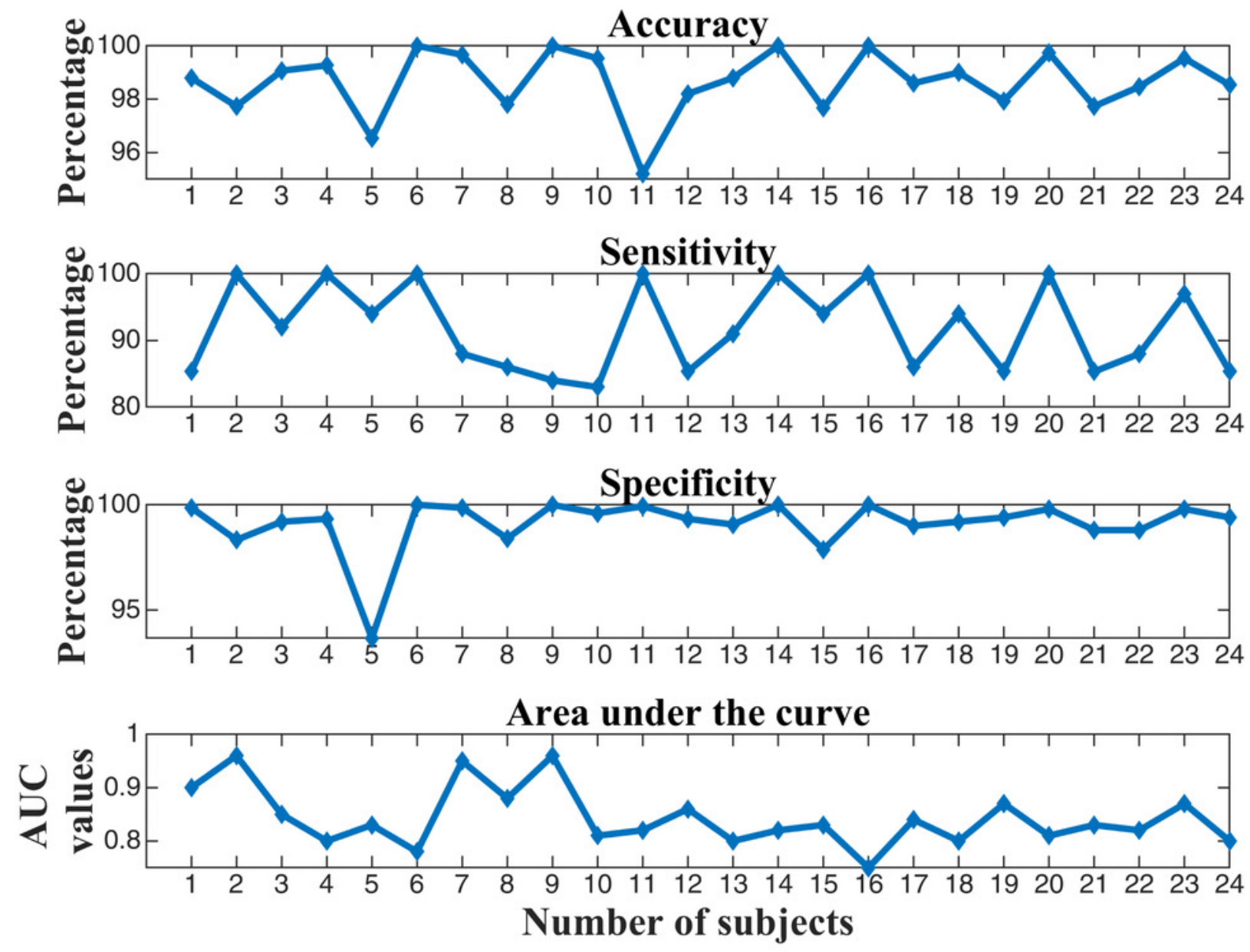
Figure 8

Performance evaluation for the prediction of EEG signals with MM-mDistEn and an ANN.

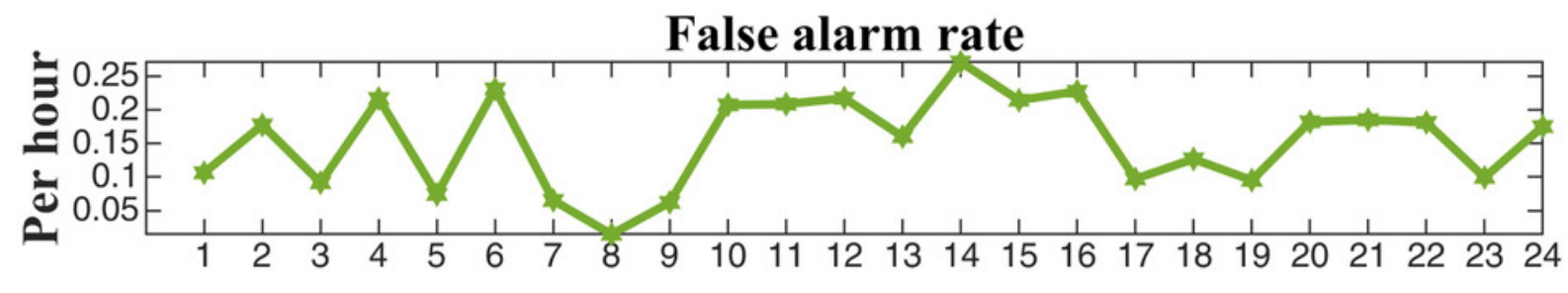

\section{Prediction time}
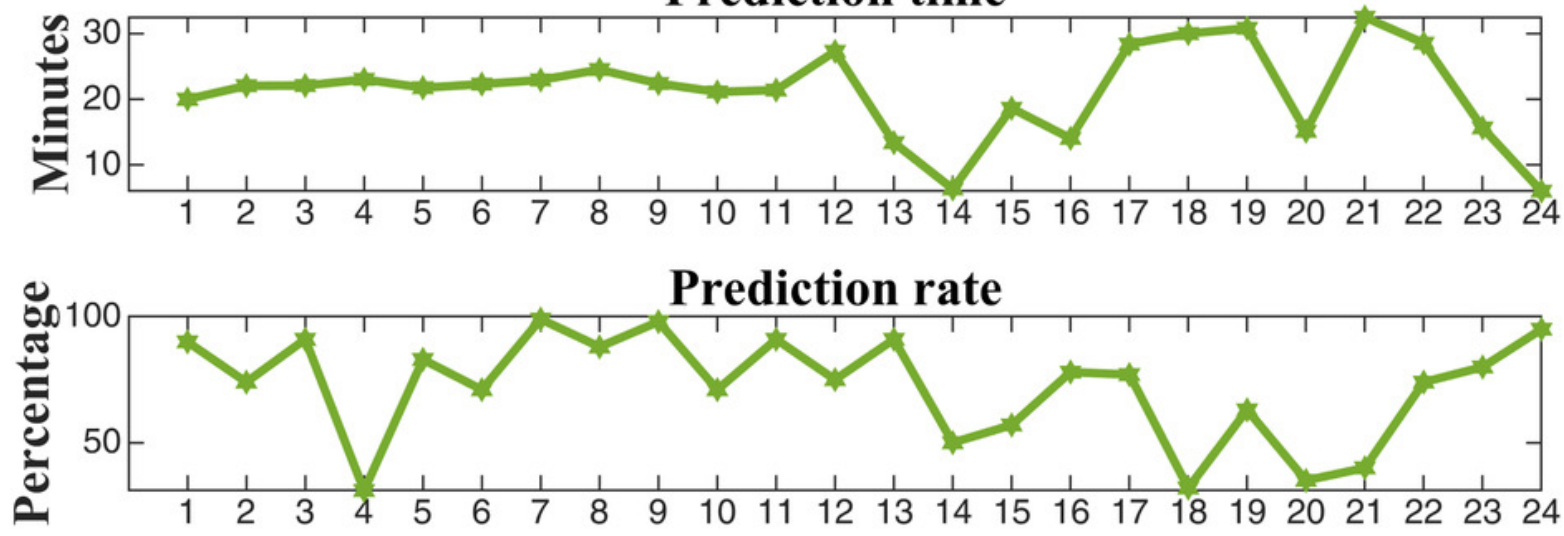

Training time

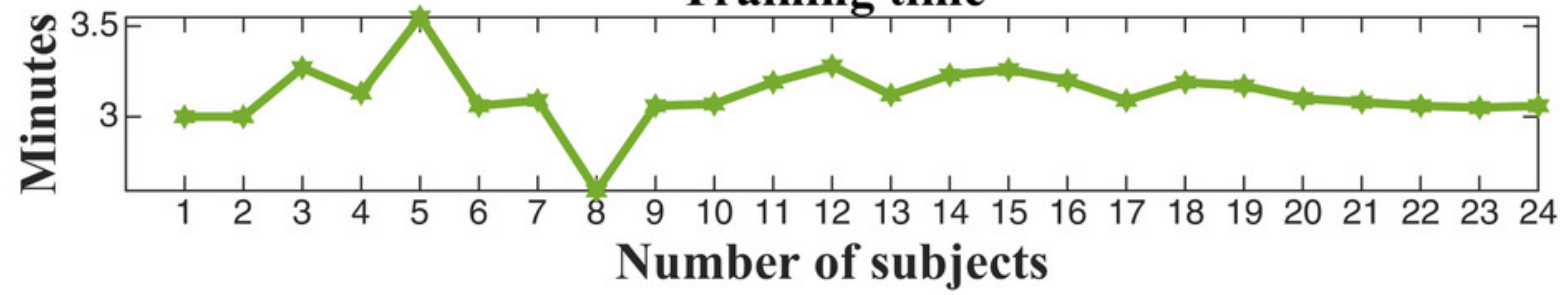




\section{Figure 9}

Prediction time and false alarm based on the different states of epileptic EEG signals.

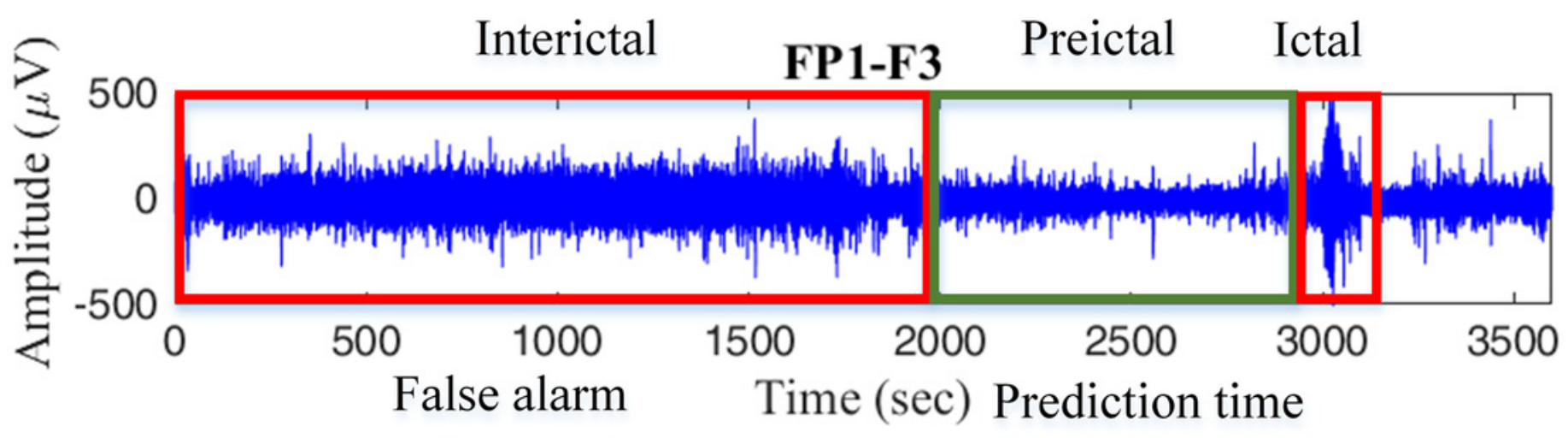




\section{Table $\mathbf{1}$ (on next page)}

Performance comparison between different prediction methods and our proposed method 
1

\begin{tabular}{|c|c|c|c|c|c|c|c|c|c|}
\hline $\begin{array}{l}\text { Feature } \\
\text { Extraction } \\
\text { Method }\end{array}$ & $\begin{array}{c}\text { Feature } \\
\text { Classification } \\
\text { Method }\end{array}$ & $\begin{array}{c}\text { Accuracy } \\
(\%)\end{array}$ & $\begin{array}{c}\text { Sensitivity } \\
(\%)\end{array}$ & $\begin{array}{l}\text { Specificity } \\
(\%)\end{array}$ & AUC & $\begin{array}{c}\text { False } \\
\text { Alarm } \\
\text { Rate } \\
\left(\mathrm{h}^{-1}\right) \\
\end{array}$ & $\begin{array}{c}\text { Prediction } \\
\text { Rate }(\%) \text { and } \\
\text { Time } \\
\text { (minutes) }\end{array}$ & $\begin{array}{l}\text { Training } \\
\text { Time } \\
\text { (minutes) }\end{array}$ & References \\
\hline $\begin{array}{c}\text { MM- } \\
\text { mDistEn }\end{array}$ & ANN & 98.66 & 91.82 & 99.11 & 0.84 & 0.014 & $99 / 26.73$ & 3.5 & - \\
\hline M-mDistEn & ANN & 88 & 85 & 90 & 0.8 & 0.081 & $90 / 23$ & 3.7 & - \\
\hline PE & ANN & 90 & 83 & 93 & 0.76 & 0.262 & $70 / 22$ & 3.5 & - \\
\hline N/A & MLP & 83.63 & 84.67 & 82.60 & N/A & 0.174 & N/A & 7.3 & (Daoud \& Bayoumi 2019) \\
\hline DCNN & MLP & 95.41 & 92.8 & 94.1 & N/A & 0.072 & N/A & 12.5 & (Daoud \& Bayoumi 2019) \\
\hline CNN & SVM & N/A & 92.7 & 90.8 & $\mathrm{~N} / \mathrm{A}$ & $\mathrm{N} / \mathrm{A}$ & $-/ 21$ & N/A & (Usman et al. 2020) \\
\hline N/A & $\mathrm{CNN}$ & 99.3 & N/A & 99.6 & N/A & 0.5 & N/A & N/A & (Gómez et al. 2020) \\
\hline
\end{tabular}

2 